\title{
AN ANALYSIS OF THE GROUP OPERATION IN UNIVERSAL TEICHMÜLLER SPACE
}

\author{
BY \\ FRED GARDINER
}

Introduction. The original motivation for this paper was to determine whether or not a certain map from universal Teichmüller space, $T$, to bounded operators in a certain Banach space was a homomorphism. It is found that this map is not homomorphic but, at the same time, precisely the extent to which it fails to be homomorphic.

The other chief results of this paper are the Theorems 1 through 5 . Theorems 1 and 2 give two descriptions of right translation which are, in a sense, dual to each other. Theorem 3 is a local description of the derivative of right translation on the tangent bundle of Teichmüller space. Its importance is that it yields a corollary which gives new reproducing formulas and it makes possible Theorem 5 which gives an explicit form of the operator $\left(\mathscr{L}_{C^{u}}^{2}\right)^{-1}$. Theorem 4 gives a geometric method of constructing inverses in Teichmüller space when points in $T$ are viewed as quasi-circles passing through 0,1 , and $\infty$. Namely, the inverse is given simply by complex conjugation.

Some of the techniques used here are fairly new and we expect that we have not exhausted all of their applications, especially to $T(G)$, Teichmüller space of the Fuchsian group $G$.

All of the results in $\S I$ are well known to people familiar with Teichmüller space theory and, in particular, the proofs of Theorems A through D and Theorem $\mathrm{C}^{\prime}$ in $\S \mathrm{V}$ can be found in the references listed in the bibliography. The numbered theorems are the author's.

I. Notation and some basic theorems. All of the results of this section are well known and the author claims responsibility for none of them.

Let $D$ be a domain in the extended complex plane, $C \cup\{\infty\}$. By $L_{\infty}(D)$ we mean the Banach space of bounded, measurable, complex-valued functions on $D$ with the essential supremum norm, \|\|$_{\infty}$. By $M(D)$ we mean the set of $\mu \in L_{\infty}(D)$, such that $\|\mu\|_{\infty}<1$. Given $\mu \in M(C)$, it is known that the Beltrami equation, $w_{\bar{z}}(z)$ $=\mu(z) w_{z}(z)$, has a global solution which is a homeomorphism of $C \cup\{\infty\}$. Moreover, this solution is unique up to linear fractional transformations, so it is completely unique if we normalize by the conditions that $w(0)=0, w(1)=1$, and $w(\infty)=\infty$. We will denote this normalized solution by $w^{\mu}$. Any solution to the

Received by the editors March 20, 1967. 
Beltrami equation is called quasi-conformal because it has a dilatation which is bounded by $\left(1+\|\mu\|_{\infty}\right) /\left(1-\|\mu\|_{\infty}\right)$.

Thus, the existence theorem for the Beltrami equation gives a one-to-one correspondence between elements of $M(C)$ and of $\Sigma^{*}(C)$, where $\Sigma^{*}(C)$ is the set of normalized quasi-conformal homeomorphisms of $C . \Sigma^{*}(C)$ has a natural topology of its own. If $w \in \Sigma^{*}(\boldsymbol{C})$, define $K(w)$ to be the maximum dilatation of $w$. Since $w$ is quasi-conformal, $K(w)$ is finite. We define a metric, $d$, on $\Sigma^{*}(C)$ by

$$
d\left(w_{1}, w_{2}\right)=\log K\left(w_{1} \circ w_{2}^{-1}\right) .
$$

THEOREM A. The correspondence $\mu \mapsto w^{\mu}$ from $M(C)$ to $\Sigma^{*}(C)$ is a homeomorphism.

This theorem is proved in [3] and [4].

We need a slightly generalized version of this theorem. We would like to replace $C$ by $D$, where $D$ is an arbitrary Jordan domain with three distinguished boundary points, which serve as normalization points for elements of $\Sigma^{*}(D)$. First, we consider the case $D=U$, the upper half plane. Let $L$ be the lower half plane. Given $\mu \in M(U), \mu^{*} \in M(C)$ is defined by

$$
\begin{aligned}
\mu^{*}(z) & =\mu(z) & & \text { for } z \in U \text { and } \\
& =j \mu(z) & & \text { for } z \in L .
\end{aligned}
$$

Here, $j \mu(z)$ is defined by $j \mu(z)=\overline{\mu(\bar{z})}$.

Note that we do not have to worry about defining $\mu^{*}$ on $R$, the real axis, or for that matter on any set of a real measure zero. Define $w_{\mu}(z)$ by setting $w_{\mu}(z)=w^{\mu *}(z)$. It is easy to show by the uniqueness of the solution to the Beltrami equation, that $j w_{\mu}(z)=w_{\mu}(z)$. Thus $w_{\mu}$ is a normalized quasi-conformal self-mapping of $C$ which preserves $R, U$, and $L$. Hence, we have shown that the map of Theorem 1 is bijective when $C$ is replaced by $U$ and $w^{\mu}$ is replaced by $w_{\mu}$. We postpone proving the map is topological until we discuss the group structure on $M(D)$.

Let $D$ be a Jordan domain. By this we mean that $D$ is a domain in $C \cup\{\infty\}$ which is connected and simply connected and bounded by a simple closed curve. Let $z_{0}, z_{1}$, and $z_{\infty}$ be three distinct points on the curve which bounds $D$ arranged in such an order that if the direction from $z_{0}$ to $z_{1}$ to $z_{\infty}$ is positive, then $D$ is the interior of the curve. Choose the unique continuous map $f$ from $U \cup \boldsymbol{R}$ onto $\bar{D}$ such that $f$ is conformal on $U$ and $f(0)=z_{0}, f(1)=z_{1}$, and $f(\infty)=z_{\infty}$. Let $\mu \in M(D)$. We wish to show that there exists a $w \in \Sigma^{*}(D)$ such that $w_{\bar{z}}(z)=\mu(z) w_{z}(z)$. We emphasize that $\Sigma^{*}(D)$ depends on the normalization. That is, $\Sigma^{*}(D)$ is the set of quasi-conformal self-mappings of $D$ which fix the points $z_{0}, z_{1}$, and $z_{\infty}$. Let

$$
\nu(z)=\mu(f(z)) \overline{f^{\prime}(z)} / f^{\prime}(z) .
$$

Then $\nu \in M(U)$ and so by the construction of the previous paragraph one can 
construct $w_{v}$. Let $w=f \circ w_{v} \circ f^{-1}$. Then $w \in \Sigma^{*}(D)$ and $w_{\xi}(\zeta)=\mu(\zeta) w_{\zeta}(\zeta)$. To see this observe that $w \circ f=f \circ w_{v}$ and

$$
\frac{w_{\bar{\xi}}(f(z)) \overline{f^{\prime}(z)}}{\left.w_{\zeta}(f(z))\right) f^{\prime}(z)}=\frac{w_{v \bar{z}}(z)}{w_{v z}(z)}=\nu(z)=\mu(f(z)) \frac{\overline{f^{\prime}(z)}}{\overline{f^{\prime}(z)}} .
$$

We denote this $w$ by $w_{\mu}$ and observe that with the normalization it is unique. Hence, we have

THEOREM B. The correspondence $\mu \mapsto w_{\mu}$ from $M(D)$ to $\Sigma^{*}(D)$ is a homeomorphism.

We have just shown that this map is defined and injective. That it is surjective is obvious because given $w \in \Sigma^{*}(D)$, let $\mu(z)=w_{\tilde{z}}(z) / w_{z}(z)$. Then $\mu \in M(D)$ and $w=w_{\mu}$.

Since composition yields a group structure on $\Sigma^{*}(D)$, a group structure is induced on $M(D)$. We shall denote the group operation by $\otimes$. $\otimes$ depends on $D$ and its distinguished boundary points. If $w_{\sigma}=w_{v} \circ w_{\mu}$, we will say that $\sigma=\nu \otimes \mu$. Using the chain rule for differentiation and the Beltrami equation, one finds that

where

$$
\nu \otimes \mu(z)=\frac{\mu(z)+\nu\left(w_{\mu}(z)\right) \theta_{\mu}(z)}{1+\overline{\mu(z)} \nu\left(w_{\mu}(z)\right) \theta_{\mu}(z)}
$$

$$
\theta_{\mu}(z)=\overline{w_{\mu z}(z)} / w_{\mu z}(z)
$$

and is a function taking values of modulus 1 . One can write $\nu$ in terms of $\mu$ and $\sigma$ in a more convenient way, namely,

$$
\nu\left(w_{\mu}(z)\right)=\frac{\sigma(z)-\mu(z)}{1-\overline{\mu(z)} \sigma(z)} \cdot \frac{1}{\theta_{\mu}(z)} .
$$

We recall that $w_{\mu}$ is close to $w_{\sigma}$ in $\Sigma^{*}(D)$ if $\log K\left(w_{\sigma} \circ w_{\mu}^{-1}\right)$ is close to zero. It is a consequence of the analysis of quasi-conformal mappings that $\log K\left(w_{v}\right)$ is close to zero if and only if $\|\nu\|$ is close to zero. Thus, from the above formula, $w_{\mu}$ is close to $w_{\sigma}$ if and only if $\mu$ is close to $\sigma$ and $\mu$ and $\sigma$ are inside the ball of radius $k<1$ contained in $M(D)$. Therefore, $\mu \mapsto w_{\mu}$ is uniformly bicontinuous on balls of radius $k<1$ in $M(D)$.

Warning. Right translation, i.e. $\nu \mapsto \nu \otimes \mu$, and taking group inverses at the origin in $M(D)$ are continuous operations. But left translation is not continuous, so $M(D)$ is not a topological group.

REMARK. If $E$ is another Jordan domain with three distinguished boundary points which are properly ordered and if $g$ is the unique continuous normalized map from $E$ to $D$ which is conformal in the interior, then $\mu(z) \rightarrow \mu(g(z))\left[j g^{\prime}(\bar{z}) / g^{\prime}(z)\right]$ from $M(D)$ onto $M(E)$ is a homeomorphism and a group isomorphism.

In order to define universal Teichmüller space, we must first make another definition. $\Sigma_{*}(D)$ is the set of quasi-conformal self-mappings of $D$ which fix 
pointwise the whole boundary of $D . \Sigma_{*}(D)$ is a closed, normal subgroup of $\Sigma^{*}(D)$. The universal Teichmüller space $T(D)$, with respect to the Jordan domain $D$, is defined as $\Sigma^{*}(D) \bmod \Sigma_{*}(D)$. It inherits the topological and complex structure from $\Sigma^{*}(D) \cong M(D)$. It can be metrized by the metric $\delta$ as follows. Let [w] and [ $\left.w^{\prime}\right]$ be equivalence classes in $T(D)$. Then

$$
\delta\left([w],\left[w^{\prime}\right]\right)=\inf d(\alpha, \beta) \quad \text { where } \alpha \in[w] \text { and } \beta \in\left[w^{\prime}\right] .
$$

In order to state the next theorem, we must make several isolated definitions. Let $C$ be a Jordan curve in the extended plane. $C$ is called a quasi-circle if and only if there is a quasi-conformal self-mapping, $w$, of the extended plane such that $w(\boldsymbol{R})=C$. It is remarkable that for any quasi-circle $C$ there is a quasi-conformal self-mapping of the extended plane $w$ such that $w(\boldsymbol{R})=C$ and $w$ is conformal in $L$. Moreover, there is the following very simple characterization of quasi-circles which is due to Ahlfors. (For a proof see [1] or [4].) Suppose $C$ is a Jordan curve which passes through $\infty$. Then $C$ is a quasi-circle if and only if $\sup |a-b| /|a-c|$ is bounded, where the supremum is taken over all $a, b$, and $c \in C$ such that $b$ is between $a$ and $c$.

Let $D$ be a Jordan domain. Let $\lambda_{D}$ be its Poincaré metric. $\lambda_{U}(z)=1 / 2 y$, where $z=x+i y$, and if $f$ is any conformal map from $U$ onto $D$, then $\lambda_{D}(f(z))\left|f^{\prime}(z)\right|=\lambda_{U}(z)$.

For any integer $q$ greater than or equal to 2 we define the Banach space $B_{q}(D)$ to be the set of all $\phi$ such that $\phi$ is holomorphic in $D, \phi(z)=O\left(|z|^{-2 q}\right)$ as $z \rightarrow \infty$ whenever $\infty \in D$, and

$$
\|\phi\|_{B_{q}(D)}=\sup _{z \in D} \lambda^{-q}(z)|\phi(z)| \text { is bounded. }
$$

The Schwarzian derivative is an operator which maps holomorphic functions into meromorphic functions defined by $f \mapsto\{f, z\}=f^{\prime \prime \prime} \mid f^{\prime}-3 / 2 \cdot f^{\prime \prime 2} / f^{\prime 2}$.

Let $\mu \in M(D)$. Define $w^{\mu}$ to be the normalized quasi-conformal self-mapping of the extended plane which satisfies $w_{\bar{z}}^{\mu}(z)=\mu(z) w_{z}^{\mu}(z)$ for $z \in D$ and $w_{\bar{z}}^{\mu}(z)=0$ for $z$ not in $D$. This means that $w^{\mu}$ is conformal in the interior of the complement of $D$. We now give a very mild generalization of a theorem of Ahlfors and Bers.

ThEOREM C (Ahlfors, Bers). Let $D_{1}$ be a domain bounded by a quasi-circle and $D_{2}$ its complementary domain. Then the map $\left[w_{\mu}\right] \mapsto\left\{w^{\mu}, z\right\}$ is well defined, bicontinuous, and biholomorphic from $T\left(D_{1}\right)$ onto a bounded open set in $B_{2}\left(D_{2}\right)$.

THEOREM D (AhLFORS, WEILL). Let $j$ be the map from holomorphic functions in $L$ to holomorphic functions in $U$ defined by $(j \phi)(z)=\overline{\phi(\bar{z})}$. Let $\gamma(\phi)=-\frac{1}{2} \lambda_{\mu}^{-2} \phi$. Then for all $\phi \in B_{2}(L)$ such that $\|\phi\|_{B_{2}(L)}<2$, we have $\left\{w^{\gamma(j \phi)}, z\right\}=\phi(z)$.

Here, $w$ is formed with respect to the domain $D=U$ and therefore is conformal in $L$.

We remark that the proof of this theorem depends on the symmetry between $L$ and $U$ and the method of proof is to write down the solution for $w$ in terms of two 
linearly independent solutions of the equation $\eta^{\prime \prime}+\frac{1}{2} \phi \eta=0$. More specifically, if $\eta_{1}$ and $\eta_{2}$ are two solutions to this equation in $L$ normalized so that

$$
\begin{array}{ll}
\eta_{1}(-i)=1, & \eta_{1}^{\prime}(-i)=0 \\
\eta_{2}(-i)=0, & \eta_{2}^{\prime}(-i)=1,
\end{array}
$$

then

$$
\begin{aligned}
w(z) & =\frac{2 i y \eta_{1}^{\prime}(\bar{z})+\eta_{1}(\bar{z})}{2 i y \eta_{2}^{\prime}(\bar{z})+\eta_{2}(\bar{z})} & & \text { for } y \geqq 0, \\
& =\frac{\eta_{1}(z)}{\eta_{2}(z)} & & \text { for } y \leqq 0 .
\end{aligned}
$$

With this remark, the proof is a simple calculation and a limit argument. It can be found in [1, p. 169], or [3], or [4].

Before ending this section, we make one more convention in notation. Let $g$ be a analytic map from a domain $D$ onto a domain $E$. Then there is an induced map $g_{p, q}^{*}$ from the space $\mathscr{F}(E)$ of functions on $E$, to the space $\mathscr{F}(D)$ of functions on $D$. Given $h \in \mathscr{F}(E), g_{p, q}^{*}(h)(z)=h(g(z)) g^{\prime}(z)^{p}\left(j g^{\prime}\right)(\bar{z})^{q}$. This is defined for all halfintegers $p$ and $q$ such that $p+q$ is an integer. If $q=0$, we abbreviate $g_{p, q}^{*}$ by $g_{p}^{*}$.

II. Description of right translation. When we speak of universal Teichmüller space $T$ without reference to a domain, we always mean $T=T(U)$ where the distinguished boundary points on the boundary of $U$ are 0,1 , and $\infty$. Similarly, when we write $w^{\mu}$ and $w_{\mu}$ without reference to a domain, we always mean that $\mu \in M(U)$ and $w^{\mu}$ and $w_{\mu}$ are normalized to fix 0,1 , and $\infty$. Because of Theorem $\mathrm{C}$, we can view $T$ as a bounded open set in $B_{2}(L)$. We adopt the notation $\left\langle w_{\mu}\right\rangle=\left\{w^{\mu}, z\right\}=\phi^{\mu}$. Our objective is to describe the map $\left\langle w_{v}\right\rangle \mapsto\left\langle w_{v} \circ w_{u}\right\rangle$ for all $\nu \in M(U)$ such that $\left\|\left\langle w_{v}\right\rangle\right\|_{B_{2}(L)}<2$.

Let $C^{\mu}=w^{\mu}(R), U^{\mu}=w^{\mu}(U)$, and $L^{\mu}=w^{\mu}(L)$. Let $f^{\mu}$ be the unique normalized conformal map from $U^{\mu}$ to $U$. Then $f^{\mu} \circ w^{\mu}=w_{\mu}$ in $U$. Let $\nu^{\#}=\left(f^{\mu}\right)_{-1,1}^{*} \nu . \nu^{\#} \in M\left(U^{\mu}\right)$ and $U^{\mu}$ has 0,1 , and $\infty$ as its distinguished boundary points.

LEMMA. If $w_{\sigma}=w_{v} \circ w_{\mu}$, then $w^{\sigma}=w^{v \#} \circ w^{\mu}$ in the extended plane and, in particular, $\left\{w^{\sigma}, z\right\}=\left\{w^{v \#} \circ w^{\mu}, z\right\}$.

Proof. Let $f^{v u}$ be the normalized conformal map from $w^{v \#}\left(U^{\mu}\right)$ onto $U$. To show that $w^{\sigma}=w^{v \#} \circ w^{\mu}$, it suffices to show that $f^{v \mu} \circ w^{v \#} \circ w^{\mu}=w_{\sigma}$ on $U$. This is sufficient because of the uniqueness of the solution to the Beltrami equation and because the equality of $w^{\sigma}$ and $w^{v \#} \circ w^{\mu}$ on $L$ will follow because both are conformal and normalized in $L$. Hence, we must show that

$$
\begin{aligned}
& f^{v \mu} \circ w^{v \#} \circ f^{\mu-1} \circ f^{\mu} \circ w^{\mu}=w_{v} \circ w_{\mu} \text { on } U, \text { i.e., } \\
& f^{v \mu} \circ w^{v \#} \circ f^{\mu-1}=w_{v}=f^{v} \circ w^{v} \text { on } U, \text { i.e., } \\
& f^{v-1} \circ f^{v \mu} \circ w^{v \#}=w^{v} \circ f^{\mu} \text { on } U^{\mu} .
\end{aligned}
$$


But, by definition, the functions in this last equation are normalized and map $U^{\mu}$ onto $U^{v}$. Therefore, to check equality it suffices to show that

$$
\frac{\left(f^{\nu-1} \circ f^{v \mu} \circ w^{v \#}\right)_{\bar{z}}}{\left(f^{v-1} \circ f^{v u} \circ w^{v \#}\right)_{z}}=\frac{\left(w^{v} \circ f^{u}\right)_{\bar{z}}}{\left(w^{v} \circ f^{\mu}\right)_{z}}
$$

But this follows from the definition of $\nu^{\#}$. Q.E.D.

This lemma generalizes to the case where $U$ is replaced by a domain bounded by a quasi-circle and by domains of more general type. For a discussion of this, see the author's thesis, entitled On right translation in Teichmüller's space (§VII).

We define a map $R_{1}$ from a domain containing the origin in $B_{2}\left(U^{u}\right)$ onto a domain containing the origin in $B_{2}\left(L^{\mu}\right)$ as follows. Let $\gamma_{1}(\phi)=-\frac{1}{2} \lambda_{U^{\mu}}^{-2} \phi$. Then let $\left(R_{1} \phi\right)=\left\{w^{\gamma_{1}(\phi)}, z\right\}$.

THEOREM 1. The map $\left\langle w_{v}\right\rangle \mapsto\left\langle w_{v} \circ w_{u}\right\rangle$ for $\left\|\left\langle w_{v}\right\rangle\right\|_{B_{2}(L)}<2$ can be described as

$$
\left\langle w_{v}\right\rangle \mapsto\left(w^{\mu}\right)_{2}^{*} R_{1}\left(f^{\mu}\right)_{2}^{*} j\left\langle w_{v}\right\rangle+\left\{w^{\mu}, z\right\} .
$$

Proof. Before beginning, we note that the Schwarzian derivative satisfies a well-known composition law called the Cayley identity. If $f$ and $g$ are two holomorphic functions such that the range of $f$ is contained in the domain of $g$, then $\{g \circ f, z\}=\{g, f\} f^{\prime}(z)^{2}+\{f, z\}$ or, equivalently, $\{g \circ f, z\}=(f)_{2}^{*}\{g, z\}+\{f, z\}$.

The map $\left\langle w_{v}\right\rangle \mapsto\left\langle w_{v} \circ w_{u}\right\rangle$ is, by definition and by the Cayley identity, the same as $\left\{w^{v}, z\right\} \mapsto\left(w^{\mu}\right)_{2}^{*}\left\{w^{v \#}, z\right\}+\left\{w^{\mu}, z\right\}$. On the other hand, the map $\left\langle w_{v}\right\rangle \mapsto\left\{w^{v \#}, z\right\}$ is the same as

$$
\begin{aligned}
\phi & \mapsto-\frac{1}{2} \lambda_{U}^{-2} j \phi=\nu \mapsto-\frac{1}{2} \lambda_{U^{\mu}}^{-2} \overline{\left(f^{\mu}\right)_{2}^{*} j \phi}=\nu^{\#} \\
& \mapsto R_{1}\left(f^{\mu}\right)_{2}^{*} j \phi .
\end{aligned}
$$

Theorem D, i.e., the fact that $\left\{w^{\gamma(j \phi)}, z\right\}=\phi(z)$, is the reason we can choose $\nu=-\frac{1}{2} \lambda_{U}^{-2} j \phi$. The fact that $\nu^{\#}$ is given by the above expression follows because $\lambda_{U}\left(f^{\mu}(\zeta)\right)\left|f^{\mu^{\prime}}(\zeta)\right|=\lambda_{U} \mu(\zeta)$. Q.E.D.

Since the derivative of $R_{1}$ at the origin is computable, this theorem makes it possible to differentiate the map $\left\langle w_{v}\right\rangle \mapsto\left\langle w_{v} \circ w_{\mu}\right\rangle$. Let $\mathscr{L}_{C^{\mu}}^{2}$ be the conjugate linear map from $B_{2}\left(U^{\mu}\right)$ to $B_{2}\left(L^{\mu}\right)$ defined by

$$
\left(\mathscr{L}_{C^{\mu}}^{2} \psi\right)(z)=c_{2} \iint_{U^{u}} \frac{\lambda_{U^{\mu}}^{-2}(\zeta) \overline{\psi(\zeta)}}{(z-\zeta)^{4}} d \xi d \eta .
$$

Here, $\zeta=\xi+i \eta$ and $c_{2}=3 / \pi$. It is known that $\mathscr{L}_{C^{\mu}}^{2}$ is the derivative at the origin of $R_{1}$. Hence, we have that the derivative of the map $\left\langle w_{v}\right\rangle \mapsto\left\langle w_{v} \circ w_{\mu}\right\rangle$ at $\left\langle w_{v}\right\rangle=0$ is

$$
\phi \mapsto\left(w^{\mu}\right)_{2}^{*} \mathscr{L}_{C^{\mu}}^{2}\left(f^{\mu}\right)_{2}^{*} j \phi,
$$

which is a linear map from $B_{2}(L)$ onto $B_{2}(L)$.

Next, we shall give a description of right translation similar to that given in 
Theorem 1. It is worth investigating because it has special significance in the case that $w_{\mu}=w_{v}^{-1}$. We will study this significance in §IV. We make the following convention: $v^{\mu}=w^{j \mu}$. This means $v^{\mu}$ is a normalized quasi-conformal self-mapping of $C$ which is $j \mu$-conformal in $L$ and conformal in $U$. Letting $h^{\mu}$ be the conformal normalized map of $v^{\mu}(L)$ onto $L$, we have that $w_{\mu}=h^{\mu} \circ v^{\mu}$, since both sides of this equation are normalized $j \mu$-conformal self-mappings of $L$.

We also define $R_{2}$ which is a map from a neighborhood containing the origin in $B_{2}\left(v^{\mu}(L)\right)$ to a neighborhood containing the origin in $B_{2}\left(v^{\mu}(U)\right)$ by

$$
\left(R_{2} \phi\right)=\left\{w^{\gamma_{2}(\phi)}, z\right\} \quad \text { where } \gamma_{2}(\phi)=-\frac{1}{2} \lambda_{D_{2}}^{-2} \phi
$$

and where $D_{2}=v^{\mu}(L)$. Then our alternate description of right translation takes the following form.

THEOREM 2. Suppose $\left\|\left\langle w_{\tau}\right\rangle\right\|_{B_{2}(L)}<2$. Then the map $\left\langle w_{\tau}\right\rangle \mapsto\left\langle w_{\tau} \circ w_{\mu}\right\rangle$ can be described as

$$
\left\langle w_{\imath}\right\rangle \mapsto j\left(v^{\mu}\right)_{2}^{*} R_{2}\left(h^{\mu}\right)_{2}^{*}\left\langle w_{\tau}\right\rangle+j\left\{v^{\mu}, z\right\} .
$$

Proof. To see this, first let $D_{1}=v^{\mu}(U), D_{2}=v^{\mu}(L)$ and let $\tau^{b}=\left(h^{\mu}\right)_{-1,1}^{*} j$ in $D_{2}$ and $\tau^{b} \equiv 0$ in $D_{1}$. Then, if one lets $v^{\tau b}$ be the normalized $\tau^{b}$-conformal self-mapping of $C$, one finds that $v^{\sigma}=v^{\tau b} \circ v^{\mu}$ whenever $w_{\sigma}=w_{\tau} \circ w_{\mu}$. The proof of this statement proceeds just as the proof of the lemma preceding Theorem 1. Hence, $\left\{v^{\sigma}, z\right\}$ $=\left(v^{\mu}\right)_{2}^{*}\left\{v^{\tau b}, z\right\}+\left\{v^{\mu}, z\right\}$ for $z \in U$. Now, we make the crucial observation that for any $\sigma \in M(U), j v^{\sigma}(z)=w^{\sigma}(z)$ for all $z \in C$ and, hence, $j\left\{v^{\sigma}, z\right\}=\left\{w^{\sigma}, z\right\}$ for all $z \in L$. Hence, if we apply $j$ to both sides of the equation for $\left\{v^{\sigma}, z\right\}$, then it is easy to arrive at this theorem after one has observed that the map

$$
\phi \mapsto-\frac{1}{2} \lambda_{L}^{-2} \phi=\tau \mapsto \tau^{b}=\left(h^{\mu}\right)_{-1,1}^{*} \tau \mapsto\left\{v^{\tau b}, z\right\}
$$

is the same as the map $\phi \mapsto R_{2}\left(h^{\mu}\right)_{2}^{*} \phi$. Q.E.D.

We wish to compute the derivative of $\left\langle w_{v}\right\rangle \mapsto\left\langle w_{v} \circ w_{\mu}\right\rangle$ at $\left\langle w_{v}\right\rangle=\left\langle w_{\tau}\right\rangle$ in order to make possible an application of the chain rule and to derive what appears to be a new class of reproducing formulas. This is the job of the next section.

\section{Differentiation of right translation at a removed point.}

THEOREM 3. The derivative of $\left\langle w_{v}\right\rangle \mapsto\left\langle w_{v} \circ w_{u}\right\rangle$ at $\left\langle w_{v}\right\rangle=\left\langle w_{\tau}\right\rangle$ when $\left\|\left\langle w_{\tau}\right\rangle\right\|_{B_{2}(L)}$ $<2$ is

$$
\phi \mapsto C_{2}\left(w^{\mu}\right)_{2}^{*} \iint_{U^{\mu}} \frac{\lambda_{U^{\mu}}^{-2}(\zeta) \phi\left(\overline{f^{\mu}}(\zeta)\right) \overline{f^{\mu \prime}}(\zeta)^{2} w_{\zeta}^{\tau \#}(\zeta)^{2} w_{z}^{\tau \#}(z)^{2} d \xi d \eta}{\left(w^{\tau \#}(\zeta)-w^{\tau^{\sharp}}(z)\right)^{4}}
$$

In this formula, $\tau^{\#}=\left(f^{\mu}\right)_{-1,1}^{*} \tau$ and $\tau=-1 / 2 \lambda_{U}^{-2} \bar{\psi}$ for some $\psi \in B_{2}(L)$ with $\|\psi\|_{B_{2}(L)}<2$. 
Proof. To compute this derivative we write the translation as a composition of maps, each of which can be differentiated. First, choose $\rho$ so that $w^{v \#}=w^{\rho} \circ w^{\imath \#}$ and then pick the following composition:

$$
\begin{aligned}
&\left\{w^{v}, z\right\} \stackrel{h_{1}}{\longmapsto} \nu \stackrel{h_{2}}{\longmapsto} \nu^{\#} \stackrel{h_{3}}{\longmapsto} \rho \stackrel{h_{4}}{\longmapsto}\left\{w^{\rho}, z\right\} \\
& \stackrel{h_{5}}{\longmapsto}\left\{w^{\rho} \circ w^{\tau^{\#}}, z\right\} \stackrel{h_{6}}{\longmapsto}\left\{w^{\nu \#} \circ w^{\mu}, z\right\} .
\end{aligned}
$$

We wish to differentiate $h_{1}$ at $\left\langle w_{v}\right\rangle=\left\langle w_{\tau}\right\rangle, h_{2}$ at $\tau, h_{3}$ at $\tau^{\#}, h_{4}$ at $0, h_{5}$ at 0 , and $h_{6}$ at $\left\{w^{\imath \#}, z\right\}$. It is easy to check that these points connect properly.

Note that $h_{2}, h_{5}$, and $h_{6}$ are either linear or linear plus constants and, thus, are trivial to differentiate. Since $\left\|\left\langle w_{\tau}\right\rangle\right\|_{B_{2}(L)}<2$, it is trivial to differentiate $h_{1}$ at $\left\langle w_{v}\right\rangle=\left\langle w_{\tau}\right\rangle$ because, by Theorem $\mathrm{D}, h_{1}(\phi)=-\frac{1}{2} \lambda_{U}^{-2} j \phi$ for $\|\phi\|_{B_{2}(L)}<2$.

Thus, we must deal with $h_{3}$ and $h_{4}$. From $w^{\text {v\# }}=w^{\rho} \circ w^{\text {ॠ\# }}$ we see that

$$
\rho\left(w^{\tau}(\zeta)\right)=\left(\frac{\nu^{\#}(\zeta)-\tau^{\#}(\zeta)}{1-\bar{\tau}^{\#}(\zeta) \nu^{\#}(\zeta)}\right) \cdot \frac{1}{\theta_{\tau}^{\#(\zeta)}} \cdot
$$

Thus the derivative of $h_{3}$ at $\tau^{\#}$ is the linear map from $L_{\infty}\left(U^{\mu}\right)$ to $L_{\infty}\left(w^{\tau \#}\left(U^{\mu}\right)\right)$ given by

$$
\sigma \mapsto\left[\frac{\sigma}{\left(1-\left|\tau^{\#}\right|^{2}\right) \theta_{\tau} \#}\right] \circ w^{\imath \#-1} .
$$

At the origin, $h_{4}$ has the derivative

$$
\sigma \mapsto c_{2} \iint_{w^{\tau^{\#}}\left(U^{\mu}\right)} \frac{\sigma(\zeta) d \xi d \eta}{(\zeta-z)^{4}}=c_{2} \iint_{U^{\mu}} \frac{\sigma\left(w^{\tau^{\sharp}}(\zeta)\right) J a c w^{\imath \#}(\zeta) d \xi d \eta}{\left(w^{\imath \#}(\zeta)-z\right)^{4}}
$$

where $J a c w^{\tau \#}(\zeta)=\left|w_{\zeta}^{\tau \#}(\zeta)\right|^{2}-\left|w_{\bar{\zeta}}^{\tau \#}(\zeta)\right|^{2}$.

Therefore,

Thus,

$$
h_{5}^{\prime} \circ h_{4}^{\prime}(\sigma)=c_{2} \iint_{U^{u}} \frac{\sigma\left(w^{\tau^{\#}}(\zeta)\right) J a c w^{\tau^{\#}}(\zeta) d \xi d \eta}{\left(w^{\tau^{\sharp}}(\zeta)-w^{\tau \#}(z)\right)^{4}} w_{z}^{\tau \#}(z)^{2} .
$$

$$
\begin{aligned}
h_{5}^{\prime} \circ h_{4}^{\prime} \circ h_{3}^{\prime} & =c_{2} \iint_{U^{\mu}} \frac{\sigma(\zeta)\left(1-\left|\tau^{\#}(\zeta)\right|^{2}\right)^{-1} \theta_{\tau} \#(\zeta)^{-1} J a c w^{\tau \#}(\zeta) d \xi d \eta}{\left(w^{\tau \#}(\zeta)-w^{\tau \#}(z)\right)^{4}} w_{z}^{\tau \#}(z)^{2} \\
& =c_{2} \iint_{U^{\mu}} \frac{\sigma(\zeta) w_{\zeta}^{\tau \#}(\zeta)^{2} w_{z}^{\tau \#}(z)^{2} d \xi d \eta}{\left(w^{\tau \#}(\zeta)-w^{\tau \#}(z)\right)^{4}}
\end{aligned}
$$

Here, we place $w_{z}^{\text {र\# }}(z)^{2}$ inside the integral to emphasize the symmetry. At this point, it is clear that the formula appearing in the statement of the theorem is correct, perhaps with a new constant, $c_{2}$.

COROLlaRY 1. If we let $w_{\mu}(z)=z$, right translation by $w_{\mu}$ is the identity, so its derivative is the identity and we find that

$$
\phi(z)=c_{2} \iint_{U} \frac{\lambda_{U}^{-2}(\zeta) \phi(\zeta) w_{\zeta}^{\tau}(\zeta)^{2} w_{z}^{\tau}(z)^{2} d \xi d \eta}{\left(w^{\tau}(\zeta)-w^{\tau}(z)\right)^{4}}
$$

for those $\tau$ of the form $\frac{1}{2} \lambda_{U}^{-2} \bar{\psi}$ where $\psi \in B_{2}(L)$ and $\|\psi\|_{B_{2}(L)}<2$. 
This formula can be derived slightly more directly by differentiating the map $\phi \mapsto\left\{w^{\gamma(j \phi)}, z\right\}$ at a point removed from the origin and then using Theorem $\mathrm{D}$ which says that this map is the identity. If we differentiate at the origin, i.e., the case where $\tau=0$, then we get a well-known formula. (See, for example, [3, Lecture 3, p. 2].)

COROLlaRY 2. Let $M_{\tau}$ denote right translation by $\left\langle w_{\tau}\right\rangle$ in Teichmüller space, i.e., $M_{\tau}\left(\left\langle w_{\sigma}\right\rangle\right)=\left\langle w_{\sigma} \circ w_{\tau}\right\rangle$. Then $M_{\tau \otimes \mu}^{\prime}(0)=M_{\mu}^{\prime}\left(\left\langle w_{\tau}\right\rangle\right) \circ M_{\tau}^{\prime}(0)$. Here, $M_{\tau \otimes \mu}^{\prime}(0)$ and $M_{\tau}^{\prime}(0)$ are given explicitly by Theorem 1 and Theorem 3 gives $M_{\mu}^{\prime}\left(\left\langle w_{\imath}\right\rangle\right)$.

Clearly, this corollary is just a statement of the chain rule for differentiation.

We have a map from Teichmüller space into invertible operators on $B_{2}(L)$ given by $\left\langle w_{\imath}\right\rangle \mapsto M_{\tau}^{\prime}(0)$. Without knowing $M_{\tau}^{\prime}(0)$ was the derivative of right translation, Professor L. Bers conjectured that this map was a representation of universal Teichmüller space, viewed as a group, i.e., $M_{\tau \otimes \mu}^{\prime}(0)=M_{\mu}^{\prime}(0) \circ M_{\tau}^{\prime}(0)$. If this were true, then $M^{\prime}(0)=M_{\mu}^{\prime}\left(\left\langle w_{\tau}\right\rangle\right)$ for all $\mu$ and all $\tau$ and $M_{\mu}$ would have a constant derivative for each $\mu$. Conceptually, it is quite clear that this cannot be true because we know that the analogous statement for right translation in $\Sigma^{*}(U)$ is false. In $\S \mathrm{V}$ we will show definitely that the conjecture was false.

IV. Right translation by the inverse. Let $\left(D_{1}, D_{2}, f, g\right)$ be a quadruple with the following properties. $D_{1}$ and $D_{2}$ are complementary Jordan domains in $C \cup\{\infty\}$ whose common boundary passes through 0,1 , and $\infty$. With respect to the Jordan curve which is their boundary and which is oriented so that the positive direction goes from 0 to 1 to $\infty, D_{1}$ is the interior. $g$ is the normalized conformal map from $L$ onto $D_{2}$ and $f$ is the normalized conformal map from $D_{1}$ onto $U . f$ and $g$ have unique continuous extensions to the boundary, which we denote by the same letters. Thus $f \circ g$ restricted to $\boldsymbol{R}$ is a normalized homeomorphism of $\boldsymbol{R}$. The following lemma is well known.

Lemma. Suppose $\left(D_{1}, D_{2}, f, g\right)$ and $\left(D_{1}^{\prime}, D_{2}^{\prime}, f^{\prime}, g^{\prime}\right)$ are two quadruples of the above type and suppose further that the Jordan curve separating $D_{1}$ and $D_{2}$ is a quasi-circle. Suppose $f^{\prime} \circ g^{\prime}|\boldsymbol{R}=f \circ g| \boldsymbol{R}$. Then $D_{1}=D_{1}^{\prime}, D_{2}=D_{2}^{\prime}, f=f^{\prime}$, and $g=g^{\prime}$. In particular, the boundary of $D_{1}^{\prime}$ is a quasi-circle.

Proof. Define a conformal mapping $h$ of $C$ onto $C$ by

$$
\begin{aligned}
h(z) & =f^{\prime-1} \circ f(z) \text { for } z \text { in the closure of } D_{1}, \text { and } \\
& =g^{\prime} \circ g^{-1}(z) \quad \text { for } z \text { in the closure of } D_{2} .
\end{aligned}
$$

It is clear that $h$ is conformal in $D_{1}$ and $D_{2}$. To see that it is well defined on the boundary, let $x$ be on the boundary and $y \in R$ such that $g(y)=x$. We want to show that $f^{\prime-1} \circ f(x)=g^{\prime} \circ g^{-1}(x)$, or that $f^{\prime-1} \circ f \circ g(y)=g^{\prime}(y)$, or that $f \circ g(y)$ $=f^{\prime} \circ g^{\prime}(y)$. But this last equation is the hypothesis. $h$ is also conformal on the quasi-circle which separates $D_{1}$ and $D_{2}$ because it has generalized derivatives there 
and one can apply Weyl's lemma. But $h$ is normalized, and hence the identity, and hence the conclusions of the lemma are obvious. Q.E.D.

Let $\mu$ and $\nu \in M(U)$ and suppose that $\nu \otimes \mu=$ (identity), i.e., $w_{v} \circ w_{\mu}(z)=z$. We recall that $w^{v}$ is the normalized quasi-conformal self-mapping of $C$ which is $\nu$ conformal in $U$ and conformal in $L$. Another way of saying this is that we extend $v$ to a function in $M(C)$ by letting $\nu \equiv 0$ in $L$. Recall, further, that $v^{\mu}=w^{j \mu}$. That is, $v^{\mu}$ is a normalized quasi-conformal self-mapping of $C$ which is $j \mu$-conformal in $L$ and conformal in $U$. Also, $h^{\mu}$ is the normalized conformal map of $v^{\mu}(L)$ onto $L$. Then on $L$ we have $w_{\mu}=h^{\mu} \circ v^{\mu}$, since both of these are $j \mu$-conformal self-mappings of $L$. Hence, restricting to $R$, we have that $v^{\mu-1} \circ h^{\mu-1}=w_{\mu}^{-1}=w_{v}=f^{\nu} \circ w^{\nu}$. Here, both $h^{\mu-1}$ and $w^{v}$ are conformal maps with domain $L$ and $v^{\mu-1}$ and $f^{v}$ are conformal maps with range $U$. Hence, the preceding lemma gives another

LEMMA. If $w_{\nu} \circ w_{\mu}(z)=z$, then $h^{\mu-1}(z)=w^{\nu}(z)$ for $z \in L$ and $v^{\mu-1}(z)=f^{\nu}(z)$ for $z$ in the domain of $f^{\nu}$.

This lemma has important consequences because it means that we can find the conformal maps $f^{\nu}$ and $h^{\mu}$ as solutions to a Beltrami equation and not just as the normalized Riemann maps of simply connected domains. Furthermore, it tells us something about the geometrical nature of the group operation in universal Teichmüller space. More specifically, observe that $T(U)$ can be viewed as the set of all quasi-circles which pass through 0,1 , and $\infty$. Let $C^{\mu}$ be the quasi-circle corresponding to the class $\left[w_{\mu}\right]$ in $T(U)$. That is, $C^{\mu}=w^{\mu}(R)$.

THEOREM 4. $\left[w_{u}\right]=\left[w_{v}\right]^{-1}$ if, and only if, $C^{u}$ is the complex conjugate of $C^{v}$ and the inverse denotes inverse with respect to the group operation on $T(U)$.

Before proving this we remark that if we took the Teichmüller space of any domain bounded by a circle, the statement of this theorem would remain the same except that we would define the bar to mean inversion with respect to the circle.

Proof. To prove the forward implication, first suppose that $w_{v} \circ w_{u}=$ (identity). Then the previous lemma implies that $v^{\mu}(\boldsymbol{R})=w^{v}(\boldsymbol{R})=C^{v}$. But $v^{\mu}=w^{j \mu}$ by definition and we have already observed in $\S \mathrm{II}$ that $j w^{\mu}=w^{j \mu}$. Thus, $v^{\mu}(\boldsymbol{R})=j w^{\mu}(\boldsymbol{R})$ which is the complex conjugate of $C^{\mu}$. It is known and easy to show that $w^{v}(R)=C^{v}$ depends only on the Teichmüller class of $w_{v}$, so we have proved the forward implication.

To see the converse, observe that the complex conjugate of $C^{v}$ is a quasi-circle and, hence, determines a $w^{\mu}$ such that $w^{\mu}(R)$ is the complex conjugate of $C^{\nu}$. We know that $w^{v}(\boldsymbol{R})=C^{v}$. Let $\sigma \otimes v=$ (identity). Then by the forward implication we have that $w^{\sigma}(R)=$ the complex conjugate of $C^{v}=C^{\mu}=w^{\mu}(R)$. Thus $\left[w_{\mu}\right]=\left[w_{\sigma}\right]$ and, hence, $\left[w_{\mu}\right]=\left[w_{v}\right]^{-1}$. Q.E.D.

This theorem is tantalizing because it indicates that there may be a geometrical way of constructing the product of two quasi-circles in $T(U)$.

Henceforth, we assume that $\left[w_{\mu}\right]=\left[w_{v}\right]^{-1}$. Using the lemma preceding Theorem 4 and the fact that quasi-circles depend only on the Teichmüller class, we know that 
$v^{\mu}(U)=$ domain $f^{\nu}$ and $w^{v}(L)=$ domain $h^{\mu}$. Let $D_{1}=v^{\mu}(U)$ and $D_{2}=w^{\nu}(L)$. We already defined the map $R_{1}$, which takes a neighborhood of the origin in $B_{2}\left(D_{1}\right)$ onto a neighborhood of the origin in $B_{2}\left(D_{2}\right)$. It is given by

$$
\left(R_{1} \phi\right)(z)=\left\{w^{\gamma_{1}(\phi)}, z\right\} \text {. }
$$

Similarly, we defined $R_{2}$ which takes a neighborhood of the origin in $B_{2}\left(D_{2}\right)$ onto a neighborhood of the origin in

$$
\left(R_{2} \phi\right)(z)=\left\{w^{\gamma_{2}(\phi)}, z\right\} .
$$

By Theorem 1, right translation by $w_{v}$ is given by

$$
\phi \mapsto\left(w^{v}\right)_{2}^{*} R_{1}\left(f^{v}\right)_{2}^{*} j \phi+\left\{w^{v}, z\right\} .
$$

Also, by Theorem 2, right translation by $w_{\mu}$ is given by

$$
\phi \mapsto j\left(v^{\mu}\right)_{2}^{*} R_{2}\left(h^{\mu}\right)_{2}^{*} \phi+j\left\{v^{\mu}, z\right\} .
$$

Hence, when we compose right translation by $w_{\mu}$ with right translation by $w_{v}$, of course, we get the identity, and the maps $R_{1}$ and $R_{2}$ are formed with respect to the same domains. So we find that

$$
\phi=\left(w^{\nu}\right)_{2}^{*} R_{1}\left(f^{\nu}\right)_{2}^{*} j\left[j\left(v^{\mu}\right)_{2}^{*} R_{2}\left(h^{\mu}\right)_{2}^{*} \phi+j\left\{v^{\mu}, z\right\}\right]+\left\{w^{v}, z\right\} .
$$

We shall differentiate this expression at $\phi=0$. In so doing, we see that we must differentiate right translation by $w_{v}$ at the point $\left\langle w_{v}^{-1}\right\rangle=j\left\{v^{\mu}, z\right\}=\left\langle w_{\mu}\right\rangle$. Thus, we can apply Theorem 3 if we assume that this element has $B_{2}(L)$-norm less than 2 . Thus, if we define

$$
M^{\prime}(\phi)(z)=c_{2} j\left(v^{\mu}\right)_{2}^{*} \iint_{L^{\nu}} \frac{\lambda_{L^{\nu}}^{-2}(\zeta) \overline{\left(\left(h^{\mu}\right)_{2}^{*} \phi\right)(\zeta)}}{(\zeta-z)^{4}} d \xi d \eta
$$

and

$$
N^{\prime}(\phi)(z)=c_{2}\left(w^{\nu}\right)_{2}^{*} \iint_{U^{v}} \frac{\lambda_{U^{v}}^{-2}(\zeta) \overline{\left(\left(f^{\nu}\right)_{2}^{*} j \phi\right)(\zeta)} w_{\zeta}^{\mu \#}(\zeta)^{2} w_{z}^{\mu \#}(z)^{2}}{\left(w^{\mu \#}(\zeta)-w^{\mu \#}(z)\right)^{4}} d \xi d \eta
$$

where $\mu^{\#}=\left(f^{\nu}\right)_{-1,1}^{*} \mu$, the chain rule yields $\left(N^{\prime} \circ M^{\prime}\right)(\phi)=\phi$. This statement has a simplification because $f^{v}=v^{\mu-1}$ on $U^{v}$ and $w^{v}=h^{\mu-1}$ on $L$. Here, $N^{\prime}$ and $M^{\prime}$ are operators on $B_{2}(L)$. Define $\mathscr{L}_{-C^{v}}^{2}: B_{2}\left(L^{v}\right) \rightarrow B_{2}\left(U^{v}\right)$ and $\mathscr{L}_{-C^{v}, w^{u}}^{2}: B_{2}\left(U^{v}\right) \rightarrow B_{2}\left(L^{v}\right)$ as follows:

$$
\begin{aligned}
\left(\mathscr{L}_{-C^{\nu}}^{2} \psi\right)(z) & =c_{2} \iint_{L^{\nu}} \frac{\lambda_{L^{\nu}}^{2}(\zeta) \overline{\psi(\zeta)} d \xi d \eta}{(\zeta-z)^{4}} \\
\left(\mathscr{L}_{C^{\nu}, w^{\mu}}^{2} \# \psi\right)(z) & =c_{2} \iint_{U^{\nu}} \frac{\lambda_{U^{\nu}}^{-2}(\zeta) \overline{\psi(\zeta)} w_{\zeta}^{\mu \#}(\zeta)^{2} w_{z}^{\mu \#}(z)^{2} d \xi d \eta}{\left(w^{\mu \#}(\zeta)-w^{\mu \#}(z)\right)^{4}} .
\end{aligned}
$$


We remark that since $\mu^{\#}=\left(f^{v}\right)_{-1,1}^{*} \mu, w^{\mu \#} \circ w^{v}(z)=z$. Thus, $w^{\mu \#}=w^{\nu-1}$ throughout the plane and $w^{\mu \#}=h^{\mu}$ in $L^{\nu}$. From the formula for $N^{\prime}$ and $M^{\prime}$, we see that

$$
\mathscr{L}_{C^{v}, w^{\mu}}^{2} \# \circ \mathscr{L}_{-C^{v}}^{2} \psi=\psi \text {. }
$$

In a similar manner, one can show that $\mathscr{L}_{-C^{v}}^{2} \circ \mathscr{L}_{C^{v}, w^{\mu}}^{2} \# \psi=\psi$. One needs only to write down the identity:

$$
\phi=j\left(v^{\mu}\right)_{2}^{*} R_{2}\left(h^{\mu}\right)_{2}^{*}\left[\left(w^{\nu}\right)_{2}^{*} R_{1}\left(f^{\nu}\right)_{2}^{*} j \phi+\left\{w^{\nu}, z\right\}\right]+j\left\{v^{\mu}, z\right\},
$$

and differentiate it at the point $\phi=\left\{w^{\mu}, z\right\}$.

THEOREM 5. $\mathscr{L}_{C^{v}, w^{\mu}}^{2} \# \circ \mathscr{L}_{-C^{\nu}}^{2}=$ identity on $B_{2}\left(L^{\nu}\right)$ and $\mathscr{L}_{-C^{\nu}}^{2} \circ \mathscr{L}_{C^{v}, w^{\mu}}^{2} \#=$ identity on $B_{2}\left(U^{v}\right)$.

A crucial step in the proof of Theorem $\mathrm{C}$ is to show that the operator $\mathscr{L}_{-C^{\nu}}^{2}$ is invertible, (see [3] or [4]). Here, we have shown that it is invertible for all $\nu \in M(U)$ such that $\left\|\langle w\rangle_{v}\right\|_{B_{2}(L)}<2$ and $\left\|\left\langle w_{v}^{-1}\right\rangle\right\|_{B_{2}(L)}<2$ and we have stated explicitly what its inverse is.

V. Specialization to a Fuchsian group. Let $G$ be a Fuchsian group. This means that $G$ is a discrete group of conformal self-mappings of $U$. We define

and

$$
\Sigma^{*}(U, G)=\left\{w_{\mu} \mid w_{\mu} A w_{\mu}^{-1} \text { is conformal for all } A \in G\right\},
$$

$$
\sum_{*}(U, G)=\left\{w_{\mu} \mid w_{\mu} \in \Sigma^{*}(U, G) \text { and } w_{\mu}(x)=x \text { for all } x \in \boldsymbol{R}\right\} .
$$

It is easy to see that the following conditions on $A$ are equivalent:

(i) $w_{\mu} A w_{\mu}^{-1}$ is conformal,

(ii) $w_{\mu} A w_{\mu}^{-1}$ is a linear fractional map from $U$ to $U$,

(iii) $\mu(A(z))\left(j A^{\prime}\right)(\bar{z}) / A^{\prime}(z)=\mu(z)$ for all $z \in U$.

Teichmüller space of the group $G$ is defined as $T(G)=\Sigma^{*}(U, G) \bmod \sum_{*}(U, G)$. A differential $q$-form with respect to $G$ is a holomorphic function $\phi$ in $U$ such that $\phi(A(z)) A^{\prime}(z)^{q}=\phi(z)$.

Let $\omega$ be a Dirichlet (fundamental) domain for the group $G$. Then

$$
\begin{aligned}
A_{q}(D, G) & =\left\{\phi \text { holomorphic in } D \mid \phi \text { is a } q \text {-form and }\|\phi\|_{A_{q}(D, G)}<\infty\right\} . \\
\|\phi\|_{A_{q}(D, G)} & =\iint_{\omega} \lambda_{D}^{2-q}(\zeta)|\phi(\zeta)| d \xi d \eta
\end{aligned}
$$

Since this integral is independent of the choice of $\omega$, sometimes we write $D / G$ in its place.

$$
\begin{gathered}
B_{q}(D, G)=\left\{\phi \text { holomorphic in } D \mid \phi \text { is a } q \text {-form and }\|\phi\|_{B_{q}(D, G)}<\infty\right\} . \\
\|\phi\|_{B_{q}(D, G)}=\|\phi\|_{B_{q}(D)}=\sup _{z \in D} \lambda_{D}^{-q}(z)|\phi(z)| . \\
C_{q}(D)=\left\{\left.\phi\left|\iint_{D} \lambda_{D}^{2-2 q}(z)\right| \phi(z)\right|^{2} d x d y=\|\phi\|_{C_{q}(D)}<\infty \text { and } \phi \text { is holomorphic in } D\right\} . \\
C_{q}(D, G)=\left\{\phi \text { holomorphic in } D \mid \phi \text { is a } q \text {-form and }\|\phi\|_{C_{q}(D, G)}<\infty\right\} \\
\|\phi\|_{C_{q}(D, G)}^{2}=\iint_{\omega} \lambda_{D}^{2-2 q}(z)|\phi(z)|^{2} d x d y .
\end{gathered}
$$


Here, again we often write $D / G$ in place of $\omega$ since the integral is independent of the choice of $\omega$.

Theorem $\mathrm{C}$ specializes to $G$ in the following way:

THEOREM $C^{\prime}$. The map $\left[w_{\mu}\right] \mapsto\left\{w^{\mu}, z\right\}$ is well defined, bicontinuous, biholomorphic from $T(G)=\Sigma^{*}(U, G) \bmod \sum_{*}(U, G)$ onto an open set in $B_{2}(L, G)$. (See [1], [3], or [4].)

If $\left[w_{\mu}\right] \in T(G)$, we write $w_{\mu} G w_{\mu}^{-1}=G_{\mu}$. The group $G_{\mu}$ depends only on the class $\left[w_{\mu}\right.$ ]. $G_{\mu}$ is again a Fuchsian group, so we can form $T\left(G_{\mu}\right)$ and we see that $\left[w_{\mu}\right]$ induces a biholomorphic map from $T\left(G_{\mu}\right)$ onto $T(G)$ via $\left[w_{v}\right] \mapsto\left[w_{v} \circ w_{\mu}\right]$. We shall need the following

Lemma. Let $\varepsilon>0$. Let $\left[w_{\mu}\right] \in T(G)$. Then there exists a finite number of groups, $G=G_{0}, G_{1}, \ldots, G_{n}=G_{\mu}$, and elements $\left[w_{\mu_{k}}\right] \in T\left(G_{k}\right)$ such that $w_{\mu_{k}} G_{k} w_{\mu_{k}}^{-1}=G_{k+1}$ for $k=0, \ldots, n-1$ and $\left\|\left\langle w_{\mu_{k}}\right\rangle\right\|_{B_{2}(L)}<\varepsilon$.

Proof. Consider the path $s \mapsto\left[w_{s u}\right]$ where $s \in[0,1]$. Given $s \in[0,1]$, there exists $\left.\delta_{s}\right\rangle 0$ such that $|t-s|\left\langle\delta_{s}\right.$ implies $\left\|\left\langle w_{t \mu} \circ w_{s \mu}^{-1}\right\rangle\right\|_{B_{2}(L)}<\varepsilon$ and $\left\|\left\langle w_{s \mu} \circ w_{t \mu}^{-1}\right\rangle\right\|_{B_{2}(L)}$ $<\varepsilon$. This is a consequence of the fact that right translation by $w_{s \mu}^{-1}$ is continuous and the maps $\sigma \mapsto\left\langle w_{\sigma}\right\rangle$ and $\sigma \mapsto\left\langle w_{\sigma}^{-1}\right\rangle$ are continuous at the origin. Thus, we have an open covering of $[0,1]$ and, by compactness, we can take a finite subcover induced by the points $s_{1}, \ldots, s_{m}$ such that $0=s_{0}<s_{1}<\cdots<s_{m}=1$. Let $I_{k}=$ $\left\{t|| t-s_{k} \mid<\delta_{s_{k}}\right\}$. Let $r_{k} \in I_{k} \cap I_{k+1}$ and $s_{k}<r_{k}<s_{k+1}$. Since $r_{k} \in I_{k}$,

$$
\left\|\left\langle w_{r_{k} \mu} \circ w_{s_{k} \mu}^{-1}\right\rangle\right\|_{B_{2}(L)}<\varepsilon .
$$

Since $r_{k} \in I_{k+1}, \|\left\langle w_{s_{k+1} 1^{\mu}} \circ w_{r_{k^{\mu}}}^{-1} \|_{B_{2}(L)}<\varepsilon\right.$. It is clear that $\left[w_{s_{k+1} \mu^{\mu}} \circ w_{r_{k^{\mu}}}^{-1}\right] \in T\left(w_{r_{k^{\mu}}} G w_{r_{k \mu}}^{-1}\right)$ and $\left[w_{r_{k} \mu} \circ w_{s_{k \mu}}^{-1}\right] \in T\left(w_{s_{k \mu}} G w_{s_{k \mu}}^{-1}\right)$. Now, a simple change of notation yields the lemma.

Roughly speaking, this lemma means that if a property of elements of $T(G)$ is true locally and if it is preserved under composition, then it is true globally.

Recall that in the notation of §III right translation by $\left\langle w_{\mu}\right\rangle$ was denoted by $M_{\mu}$. We shall now prove that $M_{\mu}$ cannot have a constant derivative for each $\mu$. As we have already remarked, this will mean that the map $\left\langle w_{\tau}\right\rangle \mapsto M_{\tau}^{\prime}(0)$ from $T(U)$ into operators on $B_{2}(L)$ cannot be homomorphic. The proof will be divided into three parts, which we now summarize:

(1) First, we will specialize to a Fuchsian group $G$ with finite area and introduce the cusp-form norm, which is a Hilbert norm. From the adjointness relation and the assumption that $M_{\mu}^{\prime}$ is constant, we will show that $M_{\mu}^{\prime}$ would be an isometry from $C_{2}(L, G)$ onto $C_{2}\left(L, w_{\mu} G w_{\mu}^{-1}\right)$.

(2) Then, we will show that the Teichmüller modular group of $T(G)$, which is formed from elements of $T(G)$ composed with linear fractional maps, would then be a group of isometries. 
(3) Lastly, we will note that a discrete group of isometries of a bounded domain in a finite dimensional Hilbert space must be compact in the sup-norm. But, it is known that the modular group is infinite and discrete for groups $G$ of finite area, and this will give the contradiction.

Part 1. The assumption that $G$ has finite area means that $\iint_{U / G} \lambda_{U}^{2}(z) d x d y<\infty$. In this case it is known that $A_{q}(L, G)=B_{q}(L, G)=C_{q}(L, G)$ for all $q \geqq 2$ and that these spaces are finite dimensional. Because of this equality, the pairing between $A_{q}(L, G)$ and $B_{q}(L, G)$ becomes a Hilbert norm, which is called the cusp-form norm. As usual, we let $G_{\mu}=w_{\mu} G w_{\mu}^{-1}$ and $G^{\mu}=w^{\mu} G w^{\mu-1}$ when $\left[w_{\mu}\right] \in T(G)$. Also, we let $U^{\mu}=w^{\mu}(U)$ and $L^{\mu}=w^{\mu}(L)$. $G^{\mu}$ is a discrete group of linear fractional transformations operating on $U^{\mu}$ and on $L^{\mu}$. Under the assumption that $M_{\mu}^{\prime}$ is constant, we wish to show that it is an isometry from $C_{2}(L, G)$ onto $C_{2}\left(L, G_{\mu}\right)$. If we prove this for arbitrary $\mu$ with $\left\|\left\langle w_{\mu}\right\rangle\right\|_{B_{2}(L)}<2$ and with $\left[w_{\mu}\right] \in T(G)$, then from the preceding lemma it will follow for all $\mu$ with $\left[w_{\mu}\right] \in T(G)$. One simply lets $\varepsilon=2$ and observes that by the chain rule and by the assumption that $M_{\mu}^{\prime}$ is constant, and the fact that a composition of isometries is an isometry, one would find that $M_{\mu}^{\prime}$ is an isometry for all $\mu$.

To see that $M_{\mu}^{\prime}$ would be an isometry, recall from $\S I$ that we can write it as a composition of maps: $M_{\mu}^{\prime}=\left(w^{\mu}\right)_{2}^{*} \mathscr{L}_{C}^{2}{ }^{\mu}\left(f^{\mu}\right)_{2}^{*} j$ where

$C_{2}(L, G) \stackrel{j}{\longrightarrow} C_{2}(U, G) \stackrel{\left(f^{\mu}\right)_{2}^{*}}{\longrightarrow} C_{2}\left(U^{\mu}, G^{\mu}\right) \stackrel{\mathscr{L}_{C^{\mu}}^{2}}{\longrightarrow} C_{2}\left(L^{\mu}, G^{\mu}\right) \stackrel{\left(w^{\mu}\right)_{2}^{*}}{\longrightarrow} C_{2}\left(L, G_{\mu}\right)$.

It is obvious that $j,\left(f^{\mu}\right)_{2}^{*}$, and $\left(w^{\mu}\right)_{2}^{*}$ are isometries in the respective cusp-form metrics. Let $w_{v} \circ w_{\mu}(z)=z$. Since $M_{\mu}$ is assumed to have constant derivative, so also does $M_{v}$. In particular, $M_{v}^{\prime}(0)=M_{v}^{\prime}\left(\left\langle w_{\mu}\right\rangle\right)$. By Theorem 3, $M_{v}^{\prime}(0)$ has the following description:

$$
\begin{aligned}
& C_{2}\left(L, G_{\mu}\right) \stackrel{\left(w^{\mu-1}\right)_{2}^{*}}{\longrightarrow} C_{2}\left(L^{\mu}, G^{\mu}\right) \stackrel{\mathscr{L}_{-C^{\mu}}^{2}}{\longrightarrow} C_{2}\left(U^{\mu}, G^{\mu}\right) \\
& \stackrel{\left(f^{\mu-1}\right)_{2}^{*}}{\longrightarrow} C_{2}(U, G) \stackrel{j}{\longrightarrow} C_{2}(L, G) .
\end{aligned}
$$

Since $M_{\mu}^{\prime}(0)^{-1}=M_{\nu}^{\prime}\left(\left\langle w_{\mu}\right\rangle\right)=M_{\nu}^{\prime}(0)$, we find after cancellation that $\mathscr{L}_{C^{\mu}}^{2} \circ \mathscr{L}_{-C^{\mu}}^{2}=I$, the identity on $C_{2}\left(L^{\mu}, G^{\mu}\right)$. But by the adjointness relation this implies that $\mathscr{L}_{C^{\mu}}^{2}$ would be an isometry, for

$$
\begin{aligned}
\|\phi\|_{C_{2}\left(U^{\mu}, G^{\mu}\right)}^{2} & =(\phi, \phi)_{2, U^{\mu} / G^{\mu}}=\left(\phi, \mathscr{L}_{-C^{\mu}}^{2} \circ \mathscr{L}_{C^{\mu}}^{2} \phi\right)_{2, U^{\mu} / G^{\mu}} \\
& =\left(\mathscr{L}_{C^{\mu}}^{2} \phi, \mathscr{L}_{C^{\mu}}^{2} \phi\right)_{2, L^{\mu} / G^{\mu}}=\left\|\mathscr{L}_{C^{\mu}}^{2} \phi\right\|_{C_{2}\left(L^{\mu}, G^{\mu}\right)}^{2} .
\end{aligned}
$$

Part 2. At this point we must define the Teichmüller modular group of $T(G)$. Consider all quasi-conformal homeomorphisms $w$ of $U$ (not necessarily normalized) such that $w G w^{-1}=G$. The modular group of $T(G)$, denoted $\bmod T(G)$, is the group of all such $w$ factored by the normal subgroup of such $w$ which satisfy $w(x)=x$ for all $x \in R$. If $[w] \in \bmod T(G)$, then there is a unique linear fractional 
self-map $A$ of $U$ and a unique $\left[w_{\mu}\right] \in T(G)$ such that $w_{\mu} \circ A=w$. In general, in fact most of the time, when $w \in \bmod T(G), w_{\mu} G w_{\mu}^{-1} \neq G$. However, we can still regard right translation by $w$ as a holomorphic map from $T(G)$ to $T(G)$; it is a composition, $T(G) \rightarrow T\left(G_{\mu}\right) \rightarrow T(G)$, where $A G_{\mu} A^{-1}=G$. It is obvious that right translation by $A$ yields an isometry from $C_{2}\left(L, G_{\mu}\right)$ to $C_{2}(L, G)$. For, if $\left[w_{v}\right] \in T\left(G_{\mu}\right)$, then $\left[A_{1} \circ w^{v} \circ A\right] \in T(G)$, where $A_{1}$ is chosen to normalize $w^{v} \circ A$, and

$$
\left\{A_{1} \circ w^{v} \circ A, z\right\}=\left\{w^{v} \circ A, z\right\}=\left\{w^{v}, A\right\} A^{\prime}(z)^{2} .
$$

Thus, if $\phi \in C_{2}\left(L, G_{\mu}\right)$, right translation by $A$ sends $\phi$ into $\phi(A z) A^{\prime}(z)^{2}$. But

$$
\begin{aligned}
\left\|\phi(A z) A^{\prime}(z)^{2}\right\|_{L / G}^{2} & =\iint_{L / G} \lambda_{L}^{-2}(z)|\phi(A z)|^{2}\left|A^{\prime}(z)\right|^{2} d x d y \\
& =\iint_{L / G_{\mu}} \lambda_{L}^{-2}(z)|\phi(z)|^{2} d x d y=\|\phi\|_{L / G_{\mu}}^{2} .
\end{aligned}
$$

Now, if the derivative of an analytic map is constantly the same linear map over an open set, then the analytic map is itself that same linear map plus a constant. This elementary theorem is true over complex Banach spaces and is a consequence of the mean-value theorem.

Thus, from Part 1 , we know that right translation by $w_{\mu}$ would be an isometry plus a constant (i.e., still isometric). We have just shown that right translation by $\boldsymbol{A}$ is an isometry. Thus, $\bmod T(G)$ would be a group of isometric self-mappings of a bounded open set in $C_{2}(L, G)$.

Part 3. Suppose $V$ is a group of isometric self-mappings of a bounded open set $T$ in $R^{n}$, where $R^{n}$ has some Hilbert norm, \|\| . If $A, B \in V$, then we define the distance from $A$ to $B$ by $d(A, B)=\sup _{x \in T}\|A x-B x\|$. Clearly, the group $V$ preserves the closure of $T$ and is isometric on the closure of $T$. Moreover, $d$ induces a topology on $V$. If $V$ is discrete, then it must be compact for let $A_{k}$ be a sequence of elements of $V$. Pick $n+1$ points in $T, x_{1}, \ldots, x_{n+1}$, such that the simplex determined by them has interior. Pick a subsequence of $A_{k}$, which we denote again by $A_{k}$, such that $\left(A_{k} x_{1}, \ldots, A_{k} x_{n+1}\right)$ converges to $\left(y_{1}, \ldots, y_{n+1}\right)$, where $y_{j} \in$ closure of $T$. Since the behavior of an isometry in $\boldsymbol{R}^{n}$ is determined by what it does on $n+1$ points which do not lie in the same hyperplane, it is obvious that $A_{k}$ converges to an isometry $A_{0}$ in the metric $d$. By discreteness, we know that $A_{k}=A_{0}$ for all but a finite number of $k$. Hence, $A_{0} \in V$ and $V$ is compact.

This completes Part 3 and gives the desired conclusion.

\section{BIBLIOGRAPHY}

1. L. Ahlfors, Lectures on quasi-conformal mapping, Van Nostrand, Princeton, N. J., 1966.

2. L. Ahlfors and L. Bers, Riemann's mapping theorem for variable metrics, Ann. of Math. 72 (1960), 385-404.

3. L. Bers, On moduli of Riemann surfaces, Notes from Eidgenossische Technische Hochschule, Zurich, summer term, 1964. 
4. L. Bers, Moduli of Riemann surfaces and Teichmüller spaces, Notes, Columbia Univ., New York, 1966.

5. R. C. Gunning, Lectures on Riemann surfaces, Princeton Univ. Press, Princeton, N. J., 1966.

6. Serge Lang, Introduction to differentiable manifolds, Interscience, New York, 1962.

7. O. Lehto and K. L. Virtanen, Quasikonforme Abbildungen, Springer, New York, 1965.

COLUmbia University,

New YoRK, New York 